\title{
Molecular Abnormalities Underlying Bone Fragility in Chronic Kidney Disease
}

\author{
Yoshiko Iwasaki, ${ }^{1}$ Junichiro James Kazama, ${ }^{2}$ and Masafumi Fukagawa ${ }^{3}$ \\ ${ }^{1}$ Department of Health Sciences, Oita University of Nursing and Health Sciences, Oita, Japan \\ ${ }^{2}$ Department of Nephrology and Hypertension, Fukushima Medical University, Fukushima, Japan \\ ${ }^{3}$ Division of Nephrology and Metabolism, Tokai University School of Medicine, Kanagawa, Japan \\ Correspondence should be addressed to Masafumi Fukagawa; fukagawa@tokai-u.jp
}

Received 31 October 2016; Revised 28 February 2017; Accepted 13 March 2017; Published 22 March 2017

Academic Editor: Wen-Chin Lee

Copyright (C) 2017 Yoshiko Iwasaki et al. This is an open access article distributed under the Creative Commons Attribution License, which permits unrestricted use, distribution, and reproduction in any medium, provided the original work is properly cited.

\begin{abstract}
Prevention of bone fractures is one goal of therapy for patients with chronic kidney disease-mineral and bone disorder (CKDMBD), as indicated by the Kidney Disease: Improving Global Outcomes guidelines. CKD patients, including those on hemodialysis, are at higher risk for fractures and fracture-related death compared to people with normal kidney function. However, few clinicians focus on this issue as it is very difficult to estimate bone fragility. Additionally, uremia-related bone fragility has a more complicated pathological process compared to osteoporosis. There are many uremia-associated factors that contribute to bone fragility, including severe secondary hyperparathyroidism, skeletal resistance to parathyroid hormone, and bone mineralization disorders. Uremia also aggravates bone volume loss, disarranges microarchitecture, and increases the deterioration of material properties of bone through abnormal bone cells or excess oxidative stress. In this review, we outline the prevalence of fractures, the interaction of CKD-MBD with osteoporosis in CKD patients, and discuss possible factors that exacerbate the mechanical properties of bone.
\end{abstract}

\section{Introduction}

Elderly people are susceptible to diseases such as hypertension, diabetes mellitus, and chronic obstructive pulmonary disease. Osteoporosis and chronic kidney disease (CKD) are also common, and the prevalence of these diseases is increasing globally, in part due to the increasing aging population. Osteoporosis under uremic conditions and management of the disease have not been widely studied. The prevalence and risk of fractures are higher in CKD patients compared to healthy people. Patients on dialysis, in particular, have an approximately fourfold greater risk for hip fractures than sex- and age-matched individuals in the general population $[1,2]$. Their fracture risk correlates positively with age, duration of dialysis, high or low parathyroid hormone (PTH) level, female gender, low body mass index, and presence of peripheral vascular calcification. Several studies report that nondialysis patients aged over 50 with estimated glomerular filtration rate (eGFR) below $60 \mathrm{~mL} / \mathrm{min} / 1.73 \mathrm{~m}^{2}$ also have a twofold greater risk for hip fractures than individuals without CKD [3-7]. A hip fracture critically limits activities of daily living and increases fracture-related mortality [8-10], and this trend is more evident in dialysis patients [11, 12]. Japanese dialysis patients, however, have relatively better prognosis with regard to survival after a hip fracture [13]. A tool called FRAX $^{\circledR}$ that can predict fracture risk appears to be useful for predicting death among Japanese hemodialysis patients [14]. Even though it remains unclear why FRAX was useful to predict mortality in Japanese dialysis patients, elucidation of the pathogenesis of decreased bone strength and treatment of fractures in patients with CKD are important to improve survival and the quality of life in this patient population. In this review, we describe the current status of fragility fractures and their treatments in CKD patients.

\section{Risk Factors of Fragility Fractures in CKD Patients}

Clinicians and researchers agree that risk factors for fractures in CKD are complicated because patients have many abnormalities that may increase fracture incidence. Advanced muscle weakness [15], frailty [16], and deteriorated cognitive 
TABLE 1: Components of bone quality.

\begin{tabular}{|c|c|c|c|}
\hline \multicolumn{2}{|c|}{ Structural properties } & \multicolumn{2}{|c|}{ Material properties } \\
\hline Analytical method & Parameter & Analytical method & Parameter \\
\hline & $\begin{array}{l}\text { Trabecular number } \\
\text { Trabecular thickness }\end{array}$ & Bone histomorphometry & Mineralization \\
\hline Bone histomorphometry & $\begin{array}{l}\text { Trabecular connectivity } \\
\text { Cortical thickness }\end{array}$ & FTIR, Raman & $\begin{array}{l}\text { Relative mineralization } \\
\text { Collagen crosslinking ration } \\
\text { Crystal size, purity, perfection }\end{array}$ \\
\hline & $\begin{array}{l}\text { Cortical thickness } \\
\text { Cortical porosity }\end{array}$ & HPLC & Collagen crosslinking type \\
\hline MicroCT, pQCT, HR-pQCT & $\begin{array}{l}\text { Trabecular number } \\
\text { Trabecular thickness }\end{array}$ & Back scattered electron imaging & Mineral density distribution \\
\hline & Trabecular connectivity & EDX & Elemental analysis \\
\hline Bone histomorphometry, CMS & Microdamage length, density & $\mathrm{X}$-ray diffraction & Apatite orientation \\
\hline
\end{tabular}

Micro-CT, microcomputed tomography; pQCT, peripheral quantitative computed tomography; HR-pQCT, high-resolution peripheral quantitative computed tomography; CMS, contact microradiograph; FTIR, Fourier transform infrared spectroscopy; HPLC, high-performance liquid chromatography; EDX, energydispersive X-ray spectroscopy.

function [17] are potential contributors to increased risk for falling among CKD patients. Falls are especially common in older CKD patients [18]. Lack of exposure to sunlight, which contributes to muscle strength, may be a risk factor, because the risk for hip fractures tends to be higher in highlatitude regions of the United States [19]. Despite the high prevalence of hip fractures, clinical studies have failed to elucidate why falling affects the risk for hip fractures but not fractures of other parts of the body such as vertebrae and wrist. In addition, CKD patients also have deteriorated mineral metabolism.

\section{Definitions of CKD-MBD, Renal Osteodystrophy, and Osteoporosis}

The three key bone lesions accompanying CKD are CKDmineral bone disorder (CKD-MBD), renal osteodystrophy, and osteoporosis, but their definitions are often ambiguous. CKD-MBD is a syndrome defined by the Kidney Disease: Improving Global Outcomes (KDIGO) guidelines as a systemic mineral metabolic disorder associated with CKD, which could result in disorders of bone metabolism and/or the cardiovascular system [20]. CKD-MBD consists of three components; abnormalities of calcium, phosphorus, PTH, and vitamin D metabolism; abnormalities in bone turnover, mineralization, volume, and strength; and soft tissue calcification including vascular calcification. This disease may manifest one component or any combination of the three. According to this definition, "renal osteodystrophy" indicates bone morphologic changes in patients with CKD and is one measure of the skeletal disorder component of CKD-MBD.

Bone lesions accompanying renal dysfunction are symptoms of CKD-MBD, but worsening of mechanical bone strength is not typically mentioned. Impairment of mechanical properties of bone comes under the term "osteoporosis," as defined by the National Institute of Health. This pathophysiology is characterized by compromised bone strength predisposing a person to increased risk of fractures [21]. In this definition, bone strength is a composite of bone mass and bone quality. Bone mass is a strong determinant of bone strength and is useful as a diagnostic tool for osteoporosis in people with extremely low bone mass. As there are no other tools to predict and/or monitor bone strength in clinical practice, bone mass measurement is considered the most informative and useful tool available to diagnosis osteoporosis. Bone mass, however, is not the only determining factor. Other factors affecting bone mechanical strength include "bone quality." Bone quality is used to describe the ability of bone to perform mechanical loadbearing functions. This definition includes all characteristics that influence the load-bearing capacity, including bone microarchitecture and material properties $[22,23]$, Table 1.

A question often arises as to which plays a more important role in bone mechanical strength, bone mass or bone quality. However, the contribution of each of the two parameters remains unclear, because several cohort studies suggest that one-half of all fragility fractures are observed in postmenopausal women with a $T$-score above $-2.5 \mathrm{SD}$, the threshold for diagnosing osteoporosis defined by the World Health Organization [24-26]. Additionally, postmenopausal women with fragility fractures had poor bone microarchitecture and altered material properties, which influence bone mechanical properties [27-29]. Therefore, bone mass measurement is strictly not the standard method for diagnosing osteoporosis.

With the progression of renal function impairment, fracture risk is remarkably high in CKD. While we suspect that osteoporosis may underlie the increased risk of fracture in $\mathrm{CKD}$, the mechanism may differ from that of primary osteoporosis characterized by marked reduction in bone mass. It is also unclear whether osteoporosis (bone fragility) associated with CKD is derived from CKD-MBD or factors other than CKD-MBD.

\section{Possible Factors Related to Weakening of Bone Strength}

Both clinical and preclinical studies suggest that loss of bone strength in CKD patients has two possible components, loss of bone mass and deterioration of bone quality. The KDIGO guidelines published in 2009 do not recommend routine 
TABLE 2: Molecular abnormalities that affect bone loss and bone quality.

\begin{tabular}{|c|c|c|c|}
\hline Category & Factor & Loss of bone mass & Deterioration of bone quality \\
\hline \multirow{5}{*}{ Humoral factors } & PTH & $\begin{array}{l}\text { Activating bone resorption and modulating } \\
\text { bone turnover }[42,43,46-50]\end{array}$ & Modulating microarchitecture [41] \\
\hline & FGF23 & Inhibiting bone formation [64] & Inhibiting mineralization [65] \\
\hline & Sclerostin & Inhibiting bone formation $[66-68,70,71,75]$ & Inhibiting mineralization [71] \\
\hline & & & Modulating material property [74] \\
\hline & Vitamin D & Inhibiting bone formation $[76,77]$ & Inhibiting mineralization [76] \\
\hline Uremia-specific & $\begin{array}{l}\text { Uremic toxins and } \\
\text { advanced oxidative stress }\end{array}$ & Modulating bone turnover [92-97] & $\begin{array}{l}\text { Modulating material property } \\
\qquad[51,83-88]\end{array}$ \\
\hline Bone aspects & $\begin{array}{l}\text { Microcrack accumulation, } \\
\text { osteocytes apoptosis }\end{array}$ & & Modulating material property [101-103] \\
\hline
\end{tabular}

Numerals are reference numbers.

bone mineral density (BMD) testing because BMD does not predict fracture risk in patients with kidney disease as it does in the general population [30]. However, a recent metaanalysis reveals that BMD is significantly lower in predialysis patients with fracture compared to those without [31]. A prospective study has shown that BMD measured by dual Xray absorptiometry (DXA) predicts incident fracture in stages 3-5 CKD patients, and the prediction ability is comparable to that using high-resolution peripheral quantitative computed tomography [32]. Furthermore, two studies have reported the assessment of BMD using DXA to predict fractures in CKD patients including those on hemodialysis $[33,34]$. Therefore, BMD measured by DXA may be useful to assess loss of bone mass or fracture risk. On the other hand, cortical bone loss that increases in advanced stage of CKD is not well depicted by DXA. Therefore, DXA still has limited clinical utility in advanced stage of CKD. More attention should be paid to other factors affecting bone strength. Factors contributing to bone strength comprising bone loss and bone quality are discussed below and summarized in Table 2 .

\section{Humoral Factors Related to Mineral Metabolism}

Progressive changes in serum biochemical parameters such as phosphorus, $\mathrm{PTH}, 1,25(\mathrm{OH})_{2}$ vitamin $\mathrm{D}_{3}$, and fibroblast growth factor 23 (FGF23) levels indicate CKD-related disturbances of mineral and endocrine factors $[35,36]$. Increased PTH levels powerfully impact bone mechanical properties, because PTH modifies the activities of bone cells, which regulate bone turnover leading to altered bone mass. PTH stimulates the osteoclastic resorption and remodeling speed, thereby increasing bone turnover. Reduction in cortical BMD and thickness together with increase in cortical porosity assessed by DXA or high-resolution peripheral quantitated tomography (HR-pQCT) have been reported to result in increased bone fragility [37-40]. In stable dialysis patients, Kazama et al. [41] showed that circulating PTH level correlates inversely with cortical porosity but not with cancellous bone volume assessed by bone histomorphometry. Parathyroidectomy in patients on maintenance hemodialysis reduced fracture risk [42]. Additionally, elevated serum alkaline phosphatase due to excessive PTH secretion is associated with higher risk of hip fracture [43]. However, contradicting results on the relationship between PTH level and structure-related bone strength have also been reported [44, 45]. Moreover, medical and surgical treatments for severe hyperactive parathyroid function have progressed, and moderate hyperparathyroidism is unlikely to be a major risk factor for skeletal fragility.

Disturbed bone remodeling (marked decreases in both bone resorption and bone formation) caused by suppressed PTH secretion or skeletal resistance to the action of PTH under uremic condition exits in low-turnover bone lesions in CKD [46-48]. This condition is called "adynamic bone," and is an increasingly common occurrence $[49,50]$. Adynamic bone constitutes $50 \%$ of all CKD-MBD in patients on peritoneal dialysis and $19 \%$ in patients on hemodialysis [30]. Several clinical and animal studies have suggested an increased fracture risk in adynamic bone disease [51-54].

To summarize, the relationship between fracture risk and PTH level, which alters bone remodeling and bone microstructure, remains controversial. Regardless of high or low PTH level, it is currently difficult to predict fracture risk by PTH level.

FGF23 is derived from osteocytes and is an endocrine hormone that regulates phosphate metabolism. FGF23 stimulates urinary phosphate excretion, suppresses absorption in the gut, and accelerates degradation of $1,25(\mathrm{OH})_{2}$ vitamin $\mathrm{D}_{3}$ in response to a high phosphate diet or a state of impaired phosphate excretion as seen in CKD. FGF23 level is elevated prior to changes in phosphate, $1,25(\mathrm{OH})_{2}$ vitamin $\mathrm{D}_{3}$, and PTH levels accompanying decline in GFR [55-57]. While some studies reported an association between elevated FGF23 secondary to early CKD and risk of fracture in elderly men with decreased eGFR [58-60], other reports found no significant relationship [61, 62]. Isakova et al. [63] analyzed 2234 subjects and reported that FGF23 level was not associated with bone loss or fracture risk in a community-based population of well-functioning older adults. A recent report found that elevated FGF23 induced by high phosphorus diet increased the expression of secreted frizzled-related protein 4 and Diccopf-1, which are Wnt signal inhibitors, and inhibited the Wnt signal pathway [64]. Another report showed that FGF23 also had a physiological role in local bone mineralization, regulating osteopontin indirectly through transcriptional control of tissue nonspecific alkaline phosphatase in a vitamin D- and klotho-independent manner [65]. These 
reports suggest that high FGF23 level may affect bone fragility by decreasing mineralization through inhibition of the Wnt pathway.

Sclerostin is a Wnt pathway inhibitor secreted by osteocytes. The canonical Wnt/ $\beta$-catenin signaling pathway directly affects osteoblast differentiation, proliferation, survival, and bone formation. Sclerostin antagonizes Wnt signaling and inactivates the pathway. The relationship between sclerostin and fracture risk is not consistent among studies [66-69]. Serum sclerostin is high in early CKD and is maintained at a high level in the advanced stages [70-73]. In an animal study, higher serum phosphate concentration derived from a high phosphorus diet was found to elevate sclerostin expression despite increased osteocyte apoptosis [74]. Combination therapy of anti-sclerostin antibody with PTH-suppressive agent was effective in improving bone mass and mechanical properties [75]. It is possible that a high sclerostin level is an aggravating factor of bone fragility.

Vitamin D $\left[25(\mathrm{OH}) \mathrm{D}_{3}\right.$ and $\left.1,25(\mathrm{OH})_{2} \mathrm{D}_{3}\right]$ deficiency and altered vitamin $\mathrm{D}$ metabolism occur in CKD patients. Because vitamin $\mathrm{D}$ is required for normal bone formation and mineralization, $25(\mathrm{OH}) \mathrm{D}_{3}$ deficiency $(<15 \mathrm{nmoL})$ is associated with less bone formation and mineralization in trabecular bone [76]. A lower vitamin D status is associated with increased fracture incidence and risk [77-80]. Recently, Murali et al. [65] showed that $1,25(\mathrm{OH})_{2} \mathrm{D}_{3}$ inhibits local mineralization by augmenting the expression of the inhibitor osteopontin. To elucidate the involvement of $1,25(\mathrm{OH})_{2} \mathrm{D}_{3}$ in bone mineralization in $\mathrm{CKD}$, further in vivo and in vitro experiments are required.

An increased incidence of bone fragility was observed in CKD irrespective of variations in $\mathrm{PTH}, 1,25(\mathrm{OH})_{2} \mathrm{D}_{3}, \mathrm{FGF} 23$, and sclerostin levels that reflect disturbances of mineral and endocrine metabolism. Factors other than CKD-MBD which may aggravate weakening of bone mechanical properties in CKD patients should be considered.

\section{Uremic Conditions Deteriorate Bone Material Properties}

Bone is composed of two organic materials, type I collagen and hydroxyapatite. The number of collagen crosslinks formed by both enzyme-induced and non-enzyme-induced processes as well as tissue mineral content (density) confer bone elasticity and strength. Various abnormalities in bone material properties are found in CKD patients.

The chemical composition of bone can be analyzed by vibrational spectroscopic methods such as Fourier transform infrared or Raman spectroscopy [81, 82]. These methods provide data on mineral parameters including the mineralto-matrix ratio (indicating the degree of mineral apposition), the degree of carbonate substituting for phosphate in the apatite lattice, and crystallinity (representing the mineral crystal size and perfection). Additionally, collagen maturity can be obtained by calculating the ratio of mature crosslinks to immature crosslinks. Alterations of these parameters in the bones have been reported in animal models of CKD [51, 83$85]$ and bone biopsy samples from hemodialysis patients $[86,87]$.
Nonphysiological collagen crosslinks formed by the actions of advanced glycation end-products are modified crosslinks and are found in increased numbers in bone biopsy samples from dialysis patients [88] and animal models of CKD [51, 83-85]. Immunostaining analysis of bones in a rat model of CKD also demonstrated increase in crosslinks modified by advanced glycation end-products and reduced lysyl oxidase protein, an enzyme required for generating physiological collagen crosslinks [89]. The degree of biological bone apatite orientation, which is related to bone elasticity, was assessed by X-ray diffraction [90] and was found to be exacerbated in a rat model of early kidney injury [84]. Interestingly, in experimental uremic animals, these changes were complicated by the progression of renal dysfunction [51], and some changes were independent of bone turnover [84]. The changes were reduced by administration of AST120 , an oral charcoal adsorbent of uremic toxins [83]. AST-120 did not change mineral metabolism. Therefore, the uremic condition may modify the material properties directly.

Uremic conditions are known to create an excess oxidative stress environment [91]. Uremic condition or a specific uremic toxin inhibits osteoblasts [92-96] and osteoclasts [97]. Although whether the material properties are altered in CKD patients with fragile bone has not been confirmed, uremia-related osteoporosis causing bone fragility should exist in CKD.

\section{Microcracks and Osteocyte Apoptosis}

Because one of the purposes of bone remodeling is to repair microdamage that occurs in bone from daily mechanical stress, suppression or absence of remodeling will result in accumulation of microdamage. Excessive accumulation of microdamage can cause fragility fractures. Although there are no reports that indicate impaired microdamage repair in lowturnover bone associated with CKD, findings that suppressed bone turnover increases fragility and fracture risk suggest accumulation of microdamage [98-100].

The rates of osteocyte apoptosis and reduced density are higher in fractured bone than in normal bone [101-103]. Empty lacunae (absence of osteocytes in lacunae) are found in renal osteodystrophy. PTH fragment, especially the cterminal PTH fragment, increases osteocyte apoptosis [104]. The c-terminal PTH fragment is accumulated in CKD, and the amount increases depending on renal insufficiency [105]. From these findings, increased osteocyte apoptosis appears to be associated with fragility fractures in CKD patients.

To summarize, bone fragility in CKD is probably caused by loss of bone mass and deterioration of bone quality through changes in blood levels of humoral factors and the presence of uremic toxins (Figure 1).

\section{Pharmacological Therapeutics for Bone Fractures in CKD Patients}

In the general population, pharmacotherapy is the mainstay of management for osteoporosis. Patients with primary osteoporosis are treated with different types of drugs. Guidelines for primary osteoporosis recommend antiresorptive drugs 


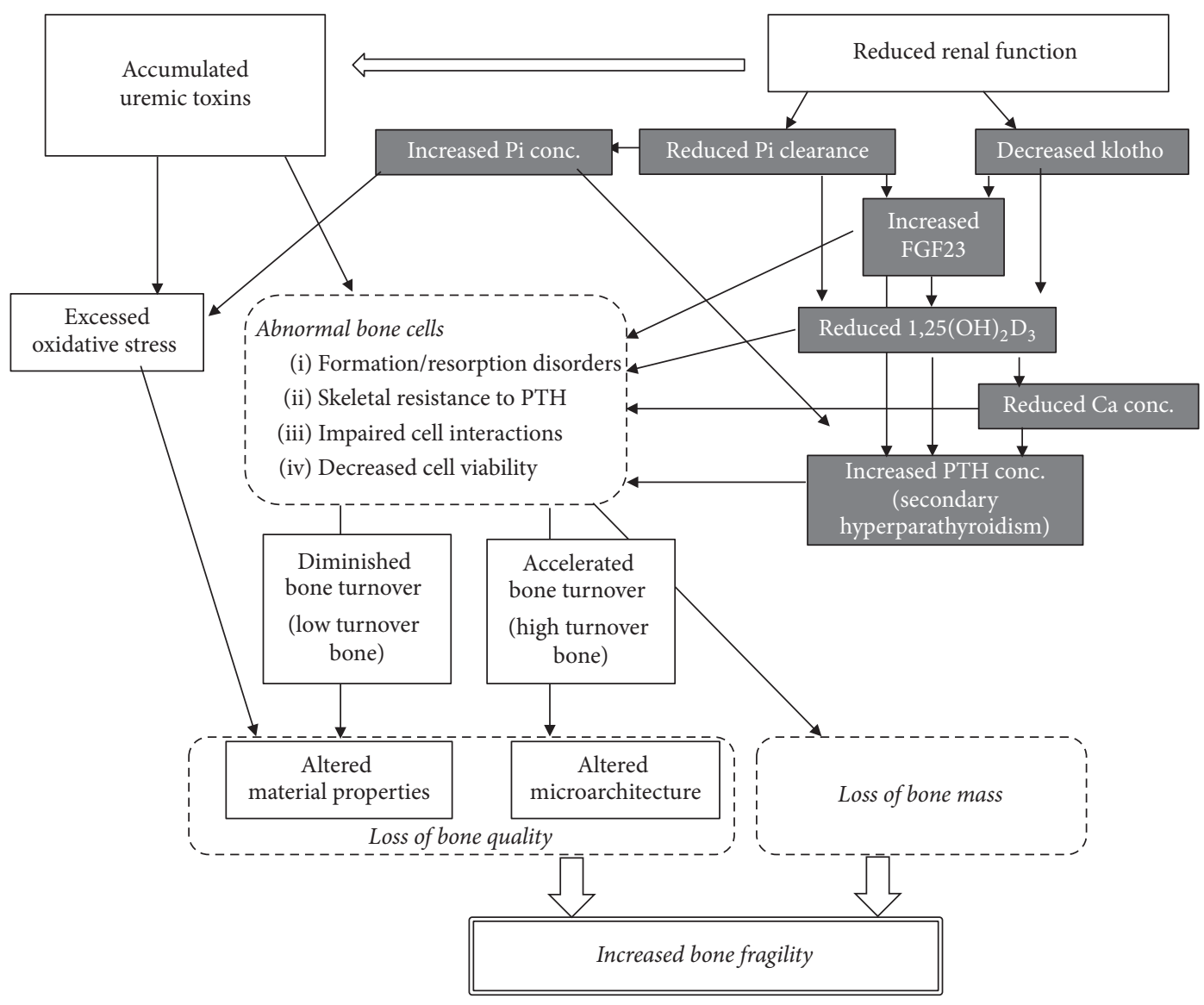

Figure 1: Possible factors involved in bone fragility. Both mineral metabolism disorders and uremic condition induce bone fragility. The detailed mechanisms and interactions are described in the text. Gray-shaded boxes indicate the phenomena induced by mineral metabolism disorders. Detailed descriptions of components of bone quality are shown in Table 1. Pi, phosphorus; Ca, calcium; FGF23, fibroblast growth factor 23; conc, concentration.

(bisphosphonates, antagonists of osteoclasts, and selective estrogen receptor modulators) and stimulators of bone formation (teriparatide) as well as active vitamin $\mathrm{D}$ and calcium supplementation. However, these drugs present problems for CKD patients, because some are excreted via the kidneys. The KDIGO guidelines [30] indicate that extrapolating results from studies of osteoporosis in general population to patients with CKD stages 3-5D may not be valid, with concerns over long-term safety because the pathogenesis differs between primary osteoporosis and CKD-MBD-related osteoporosis. On the other hand, due to the increases in osteoporosis and CKD with advancing age and the proven safety profile of osteoporotic agents, the KDIGO guidelines approve the use of these agents in early CKD patients with high risk of fracture, including patients with osteoporosis and CKD stages 1-2. Potential treatments with antiosteoporotic agents in different CKD stages are summarized in Table 3. Additional information for some agents will be discussed in detail below.

Although bisphosphonates have become a standard treatment for osteoporosis, bisphosphonates should not be used in patients with stages 4-5 CKD because these drugs are excreted by the kidney. The accumulation of bisphosphonates in bone also needs to be considered. Ott [106] reported the accumulation of bisphosphonate in the bone of dialysis patients treated with these agents. The use of bisphosphonate in dialysis is a growing concern, as there is the possibility of causing "frozen bone" with extremely low bone turnover. Bisphosphonate exposure over a 5.5-year period was reported to aggravate bone viscoelasticity and provoke atypical femoral fractures [107]. This phenomenon may be a consequence of reduced heterogeneity of material properties through suppressed bone turnover [108]. Use of bisphosphonates may increase the fracture risk through exacerbation of bone mechanical properties and increase atypical fractures [109111]. Since the degree of bisphosphonate accumulation and the efficacy of bisphosphonates both depend on their affinity to hydroxyapatite, existing data suggest treatment durations of up to 5 years with alendronate, 3 years with zoledronate, and 1 year with risedronate, although the optimal length of a "drug holiday" has not been established [112]. If it is necessary to use bisphosphonates for the management of severe osteoporosis in patients with CKD, bisphosphonates that have low affinity to hydroxyapatite crystals, such as risedronate and ibandronate, should be chosen.

Denosumab, a human monoclonal antibody for the receptor activator of nuclear factor-kappa $\mathrm{B}$, does not accumulate in the body because its point of action is limited. Its efficacy in $\mathrm{CKD}$ is expected to be the same as that in 
TABLE 3: Pharmacotherapies for osteoporosis according to stage of chronic kidney disease (CKD).

\begin{tabular}{lccc}
\hline Agents & CKD stage $\leq 3$ without biochemical abnormalities & CKD stage $>3$ with biochemical abnormalities & Dialysis (stage 5D) \\
\hline Alendronate & + & - & - \\
+ \\
Risedronate & + & - & - \\
Etidronate & - & + & + \\
Ibandronate & + & + & + \\
Minodronate & + & + & + \\
Denosumab & + & + & + \\
Raloxifene & + & + & + \\
Teriparatide & + & + \\
\hline
\end{tabular}

+: use with caution; -: avoid use.

primary osteoporosis. A previous study reported that the efficacy of denosumab, which increases BMD and suppresses fractures, did not differ depending on kidney function [113]. Another study reported that denosumab significantly increased BMD of the lumber spine and femoral neck in hemodialysis patients, although the sample size was small [114]. Denosumab may induce hypocalcemia through strong suppression of bone resorption, which tends to be amplified in CKD patients [115]. Denosumab should be prescribed with active vitamin $\mathrm{D}$ to regulate the calcium balance.

Raloxifene, a selective estrogen receptor modulator improved BMD in postmenopausal women with CKD, and greater increases in BMD were associated with lower creatinine clearance [116]. In another study, patients on raloxifene showed slower progression of kidney disorders and significantly fewer kidney-related adverse events compared to the placebo group [117]. However, reduced serum calcium concentration and increased PTH secretion were reported.

Teriparatide is a recombinant protein of PTH (1-34) and an anabolic agent for the treatment of postmenopausal osteoporosis. Although teriparatide should be used with caution in osteoporotic patients with CKD due to higher blood PTH level in secondary hyperparathyroidism associated with CKD, intermittent PTH administration can be used to induce an anabolic effect on bone in CKD. Some studies have reported that teriparatide treatment increases BMD [118-120] and ameliorates bone turnover [120]. Subjects of these studies showed decreased endogenous PTH concentration compared to appropriate controls. The effect of teriparatide treatment on CKD patients with normal or slightly higher PTH remains unknown.

Anti-sclerostin monoclonal humanized antibodies such as romosozumab and blosozumab, a new class of drugs with novel mechanisms of action, are being developed for osteoporosis treatment. In clinical trials, romosozumab and blosozumab have been shown to increase bone mass concomitant with increase in bone formation marker and decreases in bone resorption markers $[121,122]$. Increases not only in trabecular BMD but also in cortical thickness and stiffness assessed by HR-pQCT were observed in subjects taking romosozumab compared to placebo controls [123]. Although elevated levels of sclerostin have been reported in CKD stages 3 to $5 \mathrm{D}$ patients $[73,124,125]$, there are no clinical data on anti-sclerostin antibody treatment in CKD patients. In phase 2 in clinical trial of romosozumab, subjects who had estimated creatinine clearance as low as $30 \mathrm{~mL} / \mathrm{min}$ were included [121]. Since romosozumab was associated with favorable effects on bone turnover in that study population, its efficacy in improving bone fragility in CKD patients may be anticipated.

In addition to the apparent relationship between sclerostin and bone strength, blood level of sclerostin has been shown to be associated with aorta valve calcification [126] and cardiovascular mortality in CKD patients [127, 128]. Further studies are needed to investigate the efficacy of sclerostin antibody treatment not only for fracture prevention but also for reducing cardiovascular mortality in CKD patients.

Control of hyperphosphatemia is important for CKD patients to prevent cardiovascular events and reduce the risk of death. From a secondary analysis of the EVOLVE trial, cinacalcet reduced the rate of clinical fractures by 16-29\% [129]. The BONAFIDE trial demonstrated that longterm treatment with cinacalcet substantially reduced PTH and diminished elevated bone turnover as well as several biomarkers [130]. Yamamoto et al. [131] reported that dialysis patients who received angiotensin-converting enzyme inhibitors or angiotensin II type I receptor blockers had an approximately 30\% lower risk of hospitalization for any fracture. It is possible that, in addition to traditional antiosteoporotic drugs, the use of inhibitors of specific pathophysiological conditions associated with renal failure is an appropriate strategy for the treatment of osteoporosis in CKD.

\section{Conclusion}

Determining the pathogenesis of osteoporosis and treatment efficacy is difficult in CKD patients because of the complicated mineral and bone abnormalities in these patients. As described above, many factors such as BMD, humoral factors, and alterations of material properties potentially affect bone strength. However, the factors that contribute to bone strength in the setting of CKD and their mechanisms of action remain unknown. For example, no changes in structural parameters and bone mechanical parameters were observed 6 months after kidney transplantation, even though BMD was ameliorated [132]. Other report revealed that cortical porosity is not superior to BMD determined by DXA hofr identification of HD patients with fragility fracture [133]. 
Moreover, clinical assessment of human femoral mechanical properties by reference point indentation (RPI), which is a novel technique that allows direct measurement of bone material or biomechanical properties, indicated that BMD did not discriminate fracture cases form controls [134]. These recent studies suggest that bone strength in CKD patients may be affected by many factors in a complicated manner, and the major factor and its degree of contribution remain unidentified. Therefore, more studies are required to assess bone mechanical properties using a multitude of factors including BMD and humoral factors. If PRI can be used easily in clinical studies, we may be able to discuss the diagnosis or grading of bone fragility in CKD patients. CKD patients are at increased risk for fractures regardless of whether they are on dialysis. The KDIGO working group is scheduling a selective revision of the guidelines [135]. However, until then, patients at risk of fragility fractures still need to be managed. Researchers, clinicians, pharmacologists, nurses, drug companies, and other authorities should pay particular attention to osteoporosis in $\mathrm{CKD}$ patients to determine suitable management.

\section{Conflicts of Interest}

The authors declare that there are no conflicts of interest regarding the publication of this paper.

\section{References}

[1] A. M. Alem, D. J. Sherrard, D. L. Gillen et al., "Increased risk of hip fracture among patients with end-stage renal disease," Kidney International, vol. 58, no. 1, pp. 396-399, 2000.

[2] M. Coco and H. Rush, "Increased incidence of hip fractures in dialysis patients with low serum parathyroid hormone," American Journal of Kidney Diseases, vol. 36, no. 6, pp. 1115-1121, 2000.

[3] T. L. Nickolas, D. J. McMahon, and E. Shane, "Relationship between moderate to severe kidney disease and hip fracture in the United States," Journal of the American Society of Nephrology, vol. 17, no. 11, pp. 3223-3232, 2006.

[4] K. E. Ensrud, L.-Y. Lui, B. C. Taylor et al., "Renal function and risk of hip and vertebral fractures in older women," Archives of Internal Medicine, vol. 167, no. 2, pp. 133-139, 2007.

[5] S. A. Jamal, D. C. Bauer, K. E. Ensrud et al., "Alendronate treatment in women with normal to severely impaired renal function: an analysis of the fracture intervention trial," Journal of Bone and Mineral Research, vol. 22, no. 4, pp. 503-508, 2007.

[6] L. F. Fried, M. L. Biggs, M. G. Shlipak et al., "Association of kidney function with incident hip fracture in older adults," Journal of the American Society of Nephrology, vol. 18, no. 1, pp. 282-286, 2007.

[7] A. C. Dooley, N. S. Weiss, and B. Kestenbaum, "Increased risk of hip fracture among men with CKD," American Journal of Kidney Diseases, vol. 51, no. 1, pp. 38-44, 2008.

[8] A. Trombetti, F. Herrmann, P. Hoffmeyer, M. A. Schurch, J. P. Bonjour, and R. Rizzoli, "Survival and potential years of life lost after hip fracture in men and age-matched women," Osteoporosis International, vol. 13, no. 9, pp. 731-737, 2002.

[9] P. Haentjens, J. Magaziner, C. S. Colón-Emeric et al., "Metaanalysis: excess mortality after hip fracture among older women and men," Annals of Internal Medicine, vol. 152, no. 6, pp. 380390, 2010.

[10] A. Mittalhenkle, D. L. Gillen, and C. O. Stehman-Breen, "Increased risk of mortality associated with hip fracture in the dialysis population," American Journal of Kidney Diseases, vol. 44, no. 4, pp. 672-679, 2004.

[11] J. C.-F. Lin and W.-M. Liang, "Mortality and complications after hip fracture among elderly patients undergoing hemodialysis," BMC Nephrology, vol. 16, no. 1, article 100, 2015.

[12] C.-H. Kuo, T.-C. Hsieh, C.-H. Wang et al., "Increased risks of mortality and atherosclerotic complications in incident hemodialysis patients subsequently with bone fractures: a nationwide case-matched cohort study," PLoS ONE, vol. 10, no. 4, Article ID e0121705, 2015.

[13] F. Tentori, K. McCullough, R. D. Kilpatrick et al., "High rates of death and hospitalization follow bone fracture among hemodialysis patients," Kidney International, vol. 85, no. 1, pp. 166-173, 2014.

[14] T. Hayashi, N. Joki, Y. Tanaka et al., “The FRAX ${ }^{\circledR}$ as a predictor of mortality in Japanese incident hemodialysis patients: an observational, follow-up study," Journal of Bone and Mineral Metabolism, vol. 33, no. 6, pp. 674-683, 2015.

[15] P. Stenvinkel, J. J. Carrero, F. von Walden, T. A. Ikizler, and G. A. Nader, "Muscle wasting in end-stage renal disease promulgates premature death: established, emerging and potential novel treatment strategies," Nephrology Dialysis Transplantation, vol. 31, no. 7, pp. 1070-1077, 2016.

[16] C. Delgado, S. Shieh, B. Grimes et al., "Association of selfreported frailty with falls and fractures among patients new to dialysis," American Journal of Nephrology, vol. 42, no. 2, pp. 134140, 2015.

[17] M. Maravic, A. Ostertag, P. Urena, and M. Cohen-Solal, "Dementia is a major risk factor for hip fractures in patients with chronic kidney disease," Osteoporosis International, vol. 27, no. 4, pp. 1665-1669, 2016.

[18] C. Desmet, C. Beguin, C. Swine, and M. Jadoul, "Falls in hemodialysis patients: prospective study of incidence, risk factors, and complications," American Journal of Kidney Diseases, vol. 45, no. 1, pp. 148-153, 2005.

[19] J. B. Wetmore, J. Liu, H. S. Wirtz et al., "Geovariation in fracture risk among patients receiving hemodialysis," Clinical Journal of the American Society of Nephrology, vol. 11, no. 8, pp. 1413-1421, 2016.

[20] S. Moe, T. Drüeke, J. Cunningham et al., "Definition, evaluation, and classification of renal osteodystrophy: a position statement from Kidney Disease: Improving Global Outcomes (KDIGO)," Kidney International, vol. 69, no. 11, pp. 1945-1953, 2006.

[21] NIH Consensus Development Panel on Osteoporosis Prevention, Diagnosis, and Therapy, "Osteoporosis prevention, diagnosis, and therapy," The Journal of the American Medical Association, vol. 285, no. 6, pp. 785-795, 2001.

[22] M. L. Bouxsein, "Bone quality: where do we go from here?" Osteoporosis International, vol. 14, supplement 5, pp. S118-S127, 2003.

[23] D. Felsenberg and S. Boonen, "The bone quality framework: determinants of bone strength and their interrelationships, and implications for osteoporosis management," Clinical Therapeutics, vol. 27, no. 1, pp. 1-11, 2005.

[24] E. S. Siris, P. D. Miller, E. Barrett-Connor et al., "Identification and fracture outcomes of undiagnosed low bone mineral density in postmenopausal women: results from the National 
Osteoporosis Risk Assessment," Journal of the American Medical Association, vol. 286, no. 22, pp. 2815-2822, 2001.

[25] E. Sornay-Rendu, F. Munoz, P. Garnero, F. Duboeuf, and P. D. Delmas, "Identification of osteopenic women at high risk of fracture: the OFELY study," Journal of Bone and Mineral Research, vol. 20, no. 10, pp. 1813-1819, 2005.

[26] S. C. E. Schuit, M. Van Der Klift, A. E. A. M. Weel et al., "Fracture incidence and association with bone mineral density in elderly men and women: The Rotterdam Study," Bone, vol. 34, no. 1, pp. 195-202, 2004.

[27] E. M. Stein, A. Kepley, M. Walker et al., "Skeletal structure in postmenopausal women with osteopenia and fractures is characterized by abnormal trabecular plates and cortical thinning," Journal of Bone and Mineral Research, vol. 29, no. 5, pp. 11011109, 2014.

[28] Y. Bala, R. Zebaze, A. Ghasem-Zadeh et al., "Cortical porosity identifies women with osteopenia at increased risk for forearm fractures," Journal of Bone and Mineral Research, vol. 29, no. 6, pp. 1356-1362, 2014.

[29] F. Malgo, N. A. T. Hamdy, S. E. Papapoulos, and N. M. Appelman-Dijkstra, "Bone material strength as measured by microindentation in vivo is decreased in patients with fragility fractures independently of bone mineral density," Journal of Clinical Endocrinology and Metabolism, vol. 100, no. 5, pp. 20392045, 2015.

[30] Kidney Disease: Improving Global Outcomes (KDIGO) CKDMBD Work Group, "KIDGO clinical practice guideline for the diagnosis, evaluation, prevention, and treatment of chronic kidney disease-mineral bone disorder (CKD-MBD)," Kidney International, vol. 113, pp. S1-S130, 2009.

[31] R. C. Bucur, D. D. Panjwani, L. Turner, T. Rader, S. L. West, and S. A. Jamal, "Low bone mineral density and fractures in stages 3-5 CKD: an updated systematic review and meta-analysis," Osteoporosis International, vol. 26, no. 2, pp. 449-458, 2015.

[32] S. L. West, C. E. Lok, L. Langsetmo et al., "Bone mineral density predicts fractures in chronic kidney disease," Journal of Bone and Mineral Research, vol. 30, no. 5, pp. 913-919, 2015.

[33] S. Iimori, Y. Mori, W. Akita et al., "Diagnostic usefulness of bone mineral density and biochemical markers of bone turnover in predicting fracture in CKD stage 5D patients-a single-center cohort study," Nephrology Dialysis Transplantation, vol. 27, no. 1, pp. 345-351, 2012.

[34] R. H. Yenchek, J. H. Ix, M. G. Shlipak et al., "Bone mineral density and fracture risk in older individuals with CKD," Clinical Journal of the American Society of Nephrology, vol. 7, no. 7, pp. 1130-1136, 2012.

[35] S. M. Moe, T. Drüeke, N. Lameire, and G. Eknoyan, "Chronic kidney disease-mineral-bone disorder: a new paradigm," Advances in Chronic Kidney Disease, vol. 14, no. 1, pp. 3-12, 2007.

[36] M. G. Vervloet, Z. A. Massy, V. M. Brandenburg et al., "Bone: a new endocrine organ at the heart of chronic kidney disease and mineral and bone disorders," The Lancet Diabetes and Endocrinology, vol. 2, no. 5, pp. 427-436, 2014.

[37] A. M. Parfitt, "A structural approach to renal bone disease," Journal of Bone and Mineral Research, vol. 13, no. 8, pp. 12131220, 1998.

[38] S. M. Ott, "Review article: bone density in patients with chronic kidney disease stages 4-5," Nephrology, vol. 14, no. 4, pp. 395403, 2009.

[39] T. L. Nickolas, M. B. Leonard, and E. Shane, "Chronic kidney disease and bone fracture: a growing concern," Kidney International, vol. 74, no. 6, pp. 721-731, 2008.
[40] T. L. Nickolas, E. M. Stein, E. Dworakowski et al., "Rapid cortical bone loss in patients with chronic kidney disease," Journal of Bone and Mineral Research, vol. 28, no. 8, pp. 1811-1820, 2013.

[41] J. J. Kazama, R. Koda, S. Yamamoto, I. Narita, F. Gejyo, and A. Tokumoto, "Cancellous bone volume is an indicator for trabecular bone connectivity in dialysis patients," Clinical Journal of the American Society of Nephrology, vol. 5, no. 2, pp. 292-298, 2010.

[42] K. D. Rudser, I. H. de Boer, A. Dooley, B. Young, and B. Kestenbaum, "Fracture risk after parathyroidectomy among chronic hemodialysis patients," Journal of the American Society of Nephrology, vol. 18, no. 8, pp. 2401-2407, 2007.

[43] Y. Maruyama, M. Taniguchi, J. J. Kazama et al., "A higher serum alkaline phosphatase is associated with the incidence of hip fracture and mortality among patients receiving hemodialysis in Japan," Nephrology Dialysis Transplantation, vol. 29, no. 8, pp. 1532-1538, 2014.

[44] A. Trombetti, C. Stoermann, T. Chevalley et al., "Alterations of bone microstructure and strength in end-stage renal failure," Osteoporosis International, vol. 24, no. 5, pp. 1721-1732, 2013.

[45] A. L. Negri, E. E. Del Valle, M. B. Zanchetta et al., "Evaluation of bone microarchitecture by high-resolution peripheral quantitative computed tomography (HR-pQCT) in hemodialysis patients," Osteoporosis International, vol. 23, no. 10, pp. 25432550, 2012.

[46] M. Fukagawa, J. J. Kazama, and T. Shigematsu, "Skeletal resistance to PTH as a basic abnormality underlying uremic bone diseases," American Journal of Kidney Diseases, vol. 38, no. 4, supplement 1, pp. S152-S155, 2001.

[47] Y. Iwasaki, H. Yamato, T. Nii-Kono et al., "Insufficiency of PTH action on bone in uremia," Kidney International Supplements, no. 102, pp. S34-S36, 2006.

[48] J. Bover, P. Ureña, V. Brandenburg et al., "Adynamic bone disease: from bone to vessels in chronic kidney disease," Seminars in Nephrology, vol. 34, no. 6, pp. 626-640, 2014.

[49] H. H. Malluche, H. Mawad, and M.-C. Monier-Faugere, "The importance of bone health in end-stage renal disease: out of the frying pan, into the fire?" Nephrology Dialysis Transplantation, vol. 19, supplement 1, pp. i9-i13, 2004.

[50] D. J. Sherrard, G. Hercz, Y. Pei et al., "The spectrum of bone disease in end-stage renal failure-an evolving disorder," Kidney International, vol. 43, no. 2, pp. 436-442, 1993.

[51] Y. Iwasaki, J. J. Kazama, H. Yamato, and M. Fukagawa, "Changes in chemical composition of cortical bone associated with bone fragility in rat model with chronic kidney disease," Bone, vol. 48, no. 6, pp. 1260-1267, 2011.

[52] K. Atsumi, K. Kushida, K. Yamazaki, S. Shimizu, A. Ohmura, and T. Inoue, "Risk factors for vertebral fractures in renal osteodystrophy," American Journal of Kidney Diseases, vol. 33, no. 2, pp. 287-293, 1999.

[53] B. Piraino, T. Chen, L. Cooperstein, G. Segre, and J. Puschett, "Fractures and vertebral bone mineral density in patients with renal osteodystrophy," Clinical Nephrology, vol. 30, no. 2, pp. 5762, 1988.

[54] A. H. Ng, S. Omelon, F. Variola et al., "Adynamic bone decreases bone toughness during aging by affecting mineral and matrix," Journal of Bone and Mineral Research, vol. 31, no. 2, pp. 369-379, 2016.

[55] P. Evenepoel, B. Meijers, L. Viaene et al., "Fibroblast growth factor-23 in early chronic kidney disease: additional support in favor of a phosphate-centric paradigm for the pathogenesis 
of secondary hyperparathyroidism," Clinical Journal of the American Society of Nephrology, vol. 5, no. 7, pp. 1268-1276, 2010.

[56] T. Isakova, P. Wahl, G. S. Vargas et al., "Fibroblast growth factor 23 is elevated before parathyroid hormone and phosphate in chronic kidney disease," Kidney International, vol. 79, no. 12, pp. 1370-1378, 2011.

[57] C. Nakano, T. Hamano, N. Fujii et al., "Combined use of vitamin D status and FGF23 for risk stratification of renal outcome," Clinical Journal of the American Society of Nephrology, vol. 7, no. 5, pp. 810-819, 2012.

[58] M. A. I. Mirza, M. K. Karlsson, D. Mellström et al., "Serum fibroblast growth factor-23 (FGF-23) and fracture risk in elderly men," Journal of Bone and Mineral Research, vol. 26, no. 4, pp. 857-864, 2011.

[59] N. E. Lane, N. Parimi, M. Corr et al., "Association of serum fibroblast growth factor 23 (FGF23) and incident fractures in older men: the Osteoporotic Fractures in Men (MrOS) study," Journal of Bone and Mineral Research, vol. 28, no. 11, pp. 23252332, 2013.

[60] E. Kanda, M. Yoshida, and S. Sasaki, "Applicability of fibroblast growth factor 23 for evaluation of risk of vertebral fracture and chronic kidney disease-mineral bone disease in elderly chronic kidney disease patients," BMC Nephrology, vol. 13, no. 1, article 122, 2012.

[61] A. Jovanovich, P. Bùžková, M. Chonchol et al., "Fibroblast growth factor 23, bone mineral density, and risk of hip fracture among older adults: the cardiovascular health study," Journal of Clinical Endocrinology and Metabolism, vol. 98, no. 8, pp. 33233331, 2013.

[62] L. Desjardins, S. Liabeuf, C. Renard et al., "FGF23 is independently associated with vascular calcification but not bone mineral density in patients at various CKD stages," Osteoporosis International, vol. 23, no. 7, pp. 2017-2025, 2012.

[63] T. Isakova, X. Cai, J. Lee et al., "Associations of FGF23 with change in bone mineral density and fracture risk in older individuals," Journal of Bone and Mineral Research, vol. 31, no. 4, pp. 742-748, 2016.

[64] N. Carrillo-López, S. Panizo, C. Alonso-Montes et al., "Direct inhibition of osteoblastic Wnt pathway by fibroblast growth factor 23 contributes to bone loss in chronic kidney disease," Kidney International, vol. 90, no. 1, pp. 77-89, 2016.

[65] S. K. Murali, P. Roschger, U. Zeitz, K. Klaushofer, O. Andrukhova, and R. G. Erben, "FGF23 regulates bone mineralization in a $1,25(\mathrm{OH})_{2} \mathrm{D}_{3}$ and Klotho-independent manner," Journal of Bone and Mineral Research, vol. 31, no. 1, pp. 129-142, 2016.

[66] A. Arasu, P. M. Cawthon, L.-Y. Lui et al., "Serum sclerostin and risk of hip fracture in older caucasian women," Journal of Clinical Endocrinology and Metabolism, vol. 97, no. 6, pp. 20272032, 2012.

[67] M.-S. M. Ardawi, A. A. Rouzi, S. A. Al-Sibiani, N. S. Al-Senani, M. H. Qari, and S. A. Mousa, "High serum sclerostin predicts the occurrence of osteoporotic fractures in postmenopausal women: the center of excellence for osteoporosis research study," Journal of Bone and Mineral Research, vol. 27, no. 12, pp. 2592-2602, 2012.

[68] P. Garnero, E. Sornay-Rendu, F. Munoz, O. Borel, and R. D. Chapurlat, "Association of serum sclerostin with bone mineral density, bone turnover, steroid and parathyroid hormones, and fracture risk in postmenopausal women: the OFELY study," Osteoporosis International, vol. 24, no. 2, pp. 489-494, 2013.
[69] P. Szulc, C. Bertholon, O. Borel, F. Marchand, and R. Chapurlat, "Lower fracture risk in older men with higher sclerostin concentration: a prospective analysis from the MINOS study," Journal of Bone and Mineral Research, vol. 28, no. 4, pp. 855864, 2013.

[70] Y. Sabbagh, F. G. Graciolli, S. O’Brien et al., "Repression of osteocyte Wnt/ $\beta$-catenin signaling is an early event in the progression of renal osteodystrophy," Journal of Bone and Mineral Research, vol. 27, no. 8, pp. 1757-1772, 2012.

[71] D. Cejka, J. Herberth, A. J. Branscum et al., "Sclerostin and dickkopf-1 in renal osteodystrophy," Clinical Journal of the American Society of Nephrology, vol. 6, no. 4, pp. 877-882, 2011.

[72] S. Thambiah, R. Roplekar, P. Manghat et al., "Circulating sclerostin and Dickkopf-1 (DKK1) in predialysis chronic kidney disease (CKD): relationship with bone density and arterial stiffness," Calcified Tissue International, vol. 90, no. 6, pp. 473480, 2012.

[73] S. Pelletier, L. Dubourg, M.-C. Carlier, A. Hadj-Aissa, and D. Fouque, "The relation between renal function and serum sclerostin in adult patients with CKD," Clinical Journal of the American Society of Nephrology, vol. 8, no. 5, pp. 819-823, 2013.

[74] J. C. Ferreira, G. O. Ferrari, K. R. Neves et al., "Effects of dietary phosphate on adynamic bone disease in rats with chronic kidney disease-role of sclerostin?" PLoS ONE, vol. 8, no. 11, Article ID e79721, 2013.

[75] C. L. Newman, N. X. Chen, E. Smith et al., "Compromised vertebral structural and mechanical properties associated with progressive kidney disease and the effects of traditional pharmacological interventions," Bone, vol. 77, pp. 50-56, 2015.

[76] G. Coen, D. Mantella, M. Manni et al., "25-Hydroxyvitamin $\mathrm{D}$ levels and bone histomorphometry in hemodialysis renal osteodystrophy," Kidney International, vol. 68, no. 4, pp. 1840$1848,2005$.

[77] C. Ambrus, C. Almasi, K. Berta et al., "Vitamin D insufficiency and bone fractures in patients on maintenance hemodialysis," International Urology and Nephrology, vol. 43, no. 2, pp. 475482, 2011.

[78] H. A. Bischoff-Ferrari, W. C. Willett, J. B. Wong et al., "Prevention of nonvertebral fractures with oral vitamin $\mathrm{D}$ and dose dependency: a meta-analysis of randomized controlled trials," Archives of Internal Medicine, vol. 169, no. 6, pp. 551-561, 2009.

[79] R. Scragg, "Vitamin D and public health: an overview of recent research on common diseases and mortality in adulthood," Public Health Nutrition, vol. 14, no. 9, pp. 1515-1532, 2011.

[80] H. A. Bischoff-Ferrari, W. C. Willett, E. J. Orav et al., "A pooled analysis of vitamin $\mathrm{D}$ dose requirements for fracture prevention," The New England Journal of Medicine, vol. 367, no. 1, pp. 40-49, 2012.

[81] E. P. Paschalis, R. Mendelsohn, and A. L. Boskey, "Infrared assessment of bone quality: a review," Clinical Orthopaedics and Related Research, vol. 469, no. 8, pp. 2170-2178, 2011.

[82] M. D. Morris and G. S. Mandair, "Raman assessment of bone quality," Clinical Orthopaedics and Related Research, vol. 469, no. 8, pp. 2160-2169, 2011.

[83] Y. Iwasaki, J. J. Kazama, H. Yamato, H. Shimoda, and M. Fukagawa, "Accumulated uremic toxins attenuate bone mechanical properties in rats with chronic kidney disease," Bone, vol. 57, no. 2, pp. 477-483, 2013.

[84] Y. Iwasaki, J. J. Kazama, H. Yamato, A. Matsugaki, T. Nakano, and M. Fukagawa, "Altered material properties are responsible for bone fragility in rats with chronic kidney injury," Bone, vol. 81, pp. 247-254, 2015. 
[85] C. L. Newman, S. M. Moe, N. X. Chen et al., "Cortical bone mechanical properties are altered in an animal model of progressive chronic kidney disease," PLoS ONE, vol. 9, no. 6, Article ID e99262, 2014.

[86] H. H. Malluche, D. S. Porter, M.-C. Monier-Faugere, H. Mawad, and D. Pienkowski, "Differences in bone quality in low- and high-turnover renal osteodystrophy," Journal of the American Society of Nephrology, vol. 23, no. 3, pp. 525-532, 2012.

[87] H. Isaksson, M. J. Turunen, L. Rieppo et al., "Infrared spectroscopy indicates altered bone turnover and remodeling activity in renal osteodystrophy," Journal of Bone and Mineral Research, vol. 25, no. 6, pp. 1360-1366, 2010.

[88] J. Mitome, H. Yamamoto, M. Saito, K. Yokoyama, K. Marumo, and T. Hosoya, "Nonenzymatic cross-linking pentosidine increase in bone collagen and are associated with disorders of bone mineralization in dialysis patients," Calcified Tissue International, vol. 88, no. 6, pp. 521-529, 2011.

[89] C. Aoki, K. Uto, K. Honda, Y. Kato, and H. Oda, "Advanced glycation end products suppress lysyl oxidase and induce bone collagen degradation in a rat model of renal osteodystrophy," Laboratory Investigation, vol. 93, no. 11, pp. 1170-1183, 2013.

[90] T. Nakano, K. Kaibara, Y. Tabata et al., "Unique alignment and texture of biological apatite crystallites in typical calcified tissues analyzed by microbeam X-ray diffractometer system," Bone, vol. 31, no. 4, pp. 479-487, 2002.

[91] J. Himmelfarb, "Uremic toxicity, oxidative stress, and hemodialysis as renal replacement therapy," Seminars in Dialysis, vol. 22, no. 6, pp. 636-643, 2009.

[92] Y. Iwasaki-Ishizuka, H. Yamato, T. Nii-Kono, K. Kurokawa, and M. Fukagawa, "Downregulation of parathyroid hormone receptor gene expression and osteoblastic dysfunction associated with skeletal resistance to parathyroid hormone in a rat model of renal failure with low turnover bone," Nephrology Dialysis Transplantation, vol. 20, no. 9, pp. 1904-1911, 2005.

[93] J. J. Kazama, S. Yamamoto, I. Narita, and S. Kurihara, "Nuclear chromatin-concentrated osteoblasts in renal bone diseases," Therapeutic Apheresis and Dialysis, vol. 15, no. 1, pp. 9-13, 2011.

[94] T. Nii-Kono, Y. Iwasaki, M. Uchida et al., "Indoxyl sulfate induces skeletal resistance to parathyroid hormone in cultured osteoblastic cells," Kidney International, vol. 71, no. 8, pp. 738743, 2007.

[95] H. Tanaka, Y. Iwasaki, H. Yamato et al., "p-Cresyl sulfate induces osteoblast dysfunction through activating JNK and p38 MAPK pathways," Bone, vol. 56, no. 2, pp. 347-354, 2013.

[96] S. Yano, T. Yamaguchi, I. Kanazawa et al., "The uraemic toxin phenylacetic acid inhibits osteoblastic proliferation and differentiation: an implication for the pathogenesis of low turnover bone in chronic renal failure," Nephrology Dialysis Transplantation, vol. 22, no. 11, pp. 3160-3165, 2007.

[97] A. Mozar, L. Louvet, C. Godin et al., "Indoxyl sulphate inhibits osteoclast differentiation and function," Nephrology Dialysis Transplantation, vol. 27, no. 6, pp. 2176-2181, 2012.

[98] J. Bacchetta, S. Boutroy, L. Juillard et al., "Bone imaging and chronic kidney disease: will high-resolution peripheral tomography improve bone evaluation and therapeutic management?" Journal of Renal Nutrition, vol. 19, no. 1, pp. 44-49, 2009.

[99] Z. Seref-Ferlengez, O. D. Kennedy, and M. B. Schaffler, "Bone microdamage, remodeling and bone fragility: how much damage is too much damage?" BoneKEy Reports, vol. 4, article 644, 2015.
[100] H. H. Malluche, D. S. Porter, and D. Pienkowski, "Evaluating bone quality in patients with chronic kidney disease," Nature Reviews Nephrology, vol. 9, no. 11, pp. 671-680, 2013.

[101] C. R. Dunstan, N. M. Somers, and R. A. Evans, "Osteocyte death and hip fracture," Calcified Tissue International, vol. 53, supplement 1, pp. S113-S117, 1993.

[102] J. Power, B. S. Noble, N. Loveridge, K. L. Bell, N. Rushton, and J. Reeve, "Osteocyte lacunar occupancy in the femoral neck cortex: an association with cortical remodeling in hip fracture cases and controls," Calcified Tissue International, vol. 69, no. 1, pp. 13-19, 2001.

[103] J. Delgado-Calle, J. Arozamena, R. García-Renedo et al., "Osteocyte deficiency in hip fractures," Calcified Tissue International, vol. 89, no. 4, pp. 327-334, 2011.

[104] F. R. Bringhurst, "PTH receptors and apoptosis in osteocytes," Journal of Musculoskeletal Neuronal Interactions, vol. 2, no. 3, pp. 245-251, 2002.

[105] C. Donadio, M. Ardini, A. Lucchesi, E. Donadio, and T. Cantor, "Parathyroid hormone and large related C-terminal fragments increase at different rates with worsening of renal function in chronic kidney disease patients. A possible indicator of bone turnover status?" Clinical Nephrology, vol. 67, no. 3, pp. 131-139, 2007.

[106] S. M. Ott, "Bisphosphonate safety and efficacy in chronic kidney disease," Kidney International, vol. 82, no. 8, pp. 833-835, 2012.

[107] R. C. Güerri-Fernández, X. Nogués, J. M. Quesada Gómez et al., "Microindentation for in vivo measurement of bone tissue material properties in atypical femoral fracture patients and controls," Journal of Bone and Mineral Research, vol. 28, no. 1, pp. 162-168, 2013.

[108] S. Gourion-Arsiquaud, M. R. Allen, D. B. Burr, D. Vashishth, S. Y. Tang, and A. L. Boskey, "Bisphosphonate treatment modifies canine bone mineral and matrix properties and their heterogeneity," Bone, vol. 46, no. 3, pp. 666-672, 2010.

[109] E. Shane, D. Burr, B. Abrahamsen et al., "Atypical subtrochanteric and diaphyseal femoral fractures: second report of a task force of the American society for bone and mineral research," Journal of Bone and Mineral Research, vol. 29, no. 1, pp. 1-23, 2014.

[110] Z. Mahjoub, S. Jean, J.-T. Leclerc et al., "Incidence and characteristics of atypical femoral fractures: clinical and geometrical data," Journal of Bone and Mineral Research, vol. 31, no. 4, pp. 767-776, 2016.

[111] H. Sato, N. Kondo, Y. Wada et al., "The cumulative incidence of and risk factors for latent beaking in patients with autoimmune diseases taking long-term glucocorticoids and bisphosphonates," Osteoporosis International, vol. 27, no. 3, pp. 1217-1225, 2016.

[112] P. Anagnostis and J. C. Stevenson, "Bisphosphonate drug holidays-when, why and for how long?" Climacteric, vol. 18, supplement 2, pp. 32-38, 2015.

[113] S. A. Jamal, Ö. Ljunggren, C. Stehman-Breen et al., "Effects of denosumab on fracture and bone mineral density by level of kidney function," Journal of Bone and Mineral Research, vol. 26, no. 8, pp. 1829-1835, 2011.

[114] R. Hiramatsu, Y. Ubara, N. Sawa et al., "Denosumab for low bone mass in hemodialysis patients: a noncontrolled trial," American Journal of Kidney Diseases, vol. 66, no. 1, pp. 175-177, 2015.

[115] G. A. Block, H. G. Bone, L. Fang, E. Lee, and D. Padhi, "A singledose study of denosumab in patients with various degrees of 
renal impairment," Journal of Bone and Mineral Research, vol. 27, no. 7, pp. 1471-1479, 2012.

[116] A. Ishani, T. Blackwell, S. A. Jamal, S. R. Cummings, and K. E. Ensrud, "The effect of raloxifene treatment in postmenopausal women with CKD," Journal of the American Society of Nephrology, vol. 19, no. 7, pp. 1430-1438, 2008.

[117] M. L. Melamed, T. Blackwell, J. Neugarten et al., "Raloxifene, a selective estrogen receptor modulator, is renoprotective: a posthoc analysis," Kidney International, vol. 79, no. 2, pp. 241-249, 2011.

[118] K. Sumida, Y. Ubara, J. Hoshino et al., "Once-weekly teriparatide in hemodialysis patients with hypoparathyroidism and low bone mass: a prospective study," Osteoporosis International, vol. 27, no. 4, pp. 1441-1450, 2016.

[119] E. Mitsopoulos, E. Ginikopoulou, D. Economidou et al., "Impact of long-term cinacalcet, ibandronate or teriparatide therapy on bone mineral density of hemodialysis patients: a pilot study," American Journal of Nephrology, vol. 36, no. 3, pp. 238-244, 2012.

[120] D. Cejka, K. Kodras, T. Bader, and M. Haas, "Treatment of hemodialysis-associated adynamic bone disease with teriparatide (PTH1-34): a pilot study," Kidney and Blood Pressure Research, vol. 33, no. 3, pp. 221-226, 2010.

[121] M. R. McClung, A. Grauer, S. Boonen et al., "Romosozumab in postmenopausal women with low bone mineral density," The New England Journal of Medicine, vol. 370, no. 5, pp. 412-420, 2014.

[122] C. P. Recknor, R. R. Recker, C. T. Benson et al., "The effect of discontinuing treatment with blosozumab: follow-up results of a phase 2 randomized clinical trial in postmenopausal women with low bone mineral density," Journal of Bone and Mineral Research, vol. 30, no. 9, pp. 1717-1725, 2015.

[123] C. Graeff, G. M. Campbell, J. Peña et al., "Administration of romosozumab improves vertebral trabecular and cortical bone as assessed with quantitative computed tomography and finite element analysis," Bone, vol. 81, pp. 364-369, 2015.

[124] D. Cejka, R. Marculescu, N. Kozakowski et al., "Renal elimination of sclerostin increases with declining kidney function," The Journal of Clinical Endocrinology \& Metabolism, vol. 99, no. 1, pp. 248-255, 2014.

[125] L. Desjardins, S. Liabeuf, R. B. Oliveira et al., "Uremic toxicity and sclerostin in chronic kidney disease patients," Nephrologie et Therapeutique, vol. 10, no. 6, pp. 463-470, 2014.

[126] R. Koos, V. Brandenburg, A. H. Mahnken et al., "Sclerostin as a potential novel biomarker for aortic valve calcification: an invivo and ex-vivo study," The Journal of Heart Valve Disease, vol. 22, no. 3, pp. 317-325, 2013.

[127] M. Kanbay, D. Siriopol, M. Saglam et al., "Serum sclerostin and adverse outcomes in nondialyzed chronic kidney disease patients," The Journal of Clinical Endocrinology \& Metabolism, vol. 99, no. 10, pp. E1854-E1861, 2014.

[128] C. Drechsler, P. Evenepoel, M. G. Vervloet et al., "High levels of circulating sclerostin are associated with better cardiovascular survival in incident dialysis patients: results from the NECOSAD study," Nephrology Dialysis Transplantation, vol. 30, no. 2, pp. 288-293, 2015.

[129] S. M. Moe, S. Abdalla, G. M. Chertow et al., "Effects of cinacalcet on fracture events in patients receiving hemodialysis: the EVOLVE trial," Journal of the American Society of Nephrology, vol. 26, no. 6, pp. 1466-1475, 2015.

[130] G. J. Behets, G. Spasovski, L. R. Sterling et al., "Bone histomorphometry before and after long-term treatment with cinacalcet in dialysis patients with secondary hyperparathyroidism," Kidney International, vol. 87, no. 4, pp. 846-856, 2015.

[131] S. Yamamoto, R. Kido, Y. Onishi et al., "Use of renin-angiotensin system inhibitors is associated with reduction of fracture risk in hemodialysis patients," PLoS ONE, vol. 10, no. 4, Article ID e0122691, 2015.

[132] C. S. Rajapakse, M. B. Leonard, Y. A. Bhagat, W. Sun, J. F. Magland, and F. W. Wehrli, "Micro-MR imaging-based computational biomechanics demonstrates reduction in cortical and trabecular bone strength after renal transplantation," Radiology, vol. 262, no. 3, pp. 912-920, 2012.

[133] B. Bielesz, J. M. Patsch, L. Fischer et al., "Cortical porosity not superior to conventional densitometry in identifying hemodialysis patients with fragility fracture," PLoS ONE, vol. 12, no. 2, Article ID e0171873, 2017.

[134] T. Jenkins, L. V. Coutts, S. D’Angelo et al., "Site-dependent reference point microindentation complements clinical measures for improved fracture risk assessment at the human femoral neck," Journal of Bone and Mineral Research, vol. 31, no. 1, pp. 196-203, 2016.

[135] M. Ketteler, G. J. Elder, P. Evenepoel et al., "Revisiting KDIGO clinical practice guideline on chronic kidney disease-mineral and bone disorder: a commentary from a Kidney Disease: improving Global Outcomes controversies conference," Kidney International, vol. 87, no. 3, pp. 502-508, 2015. 


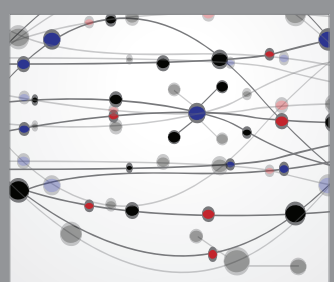

The Scientific World Journal
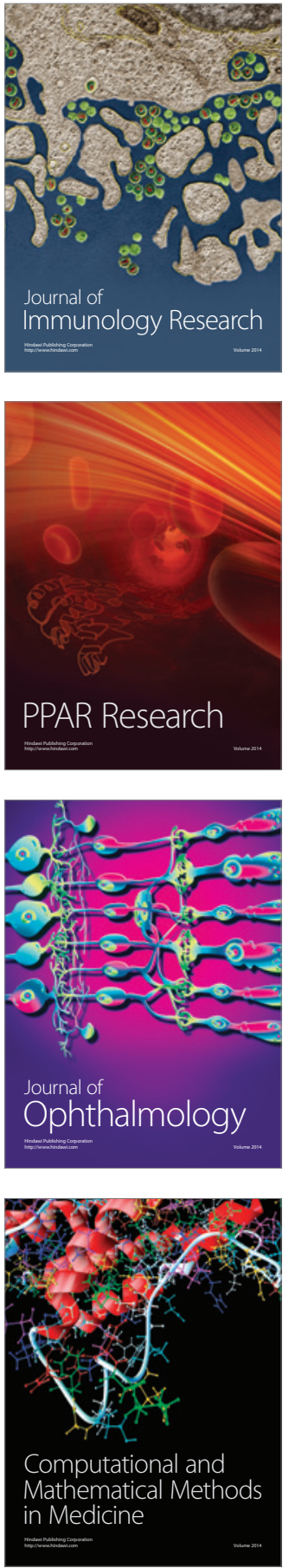

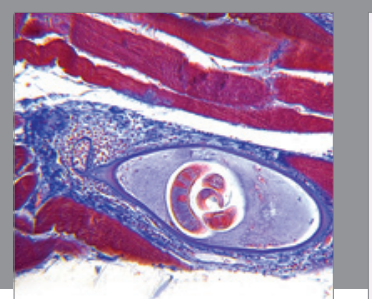

Gastroenterology Research and Practice
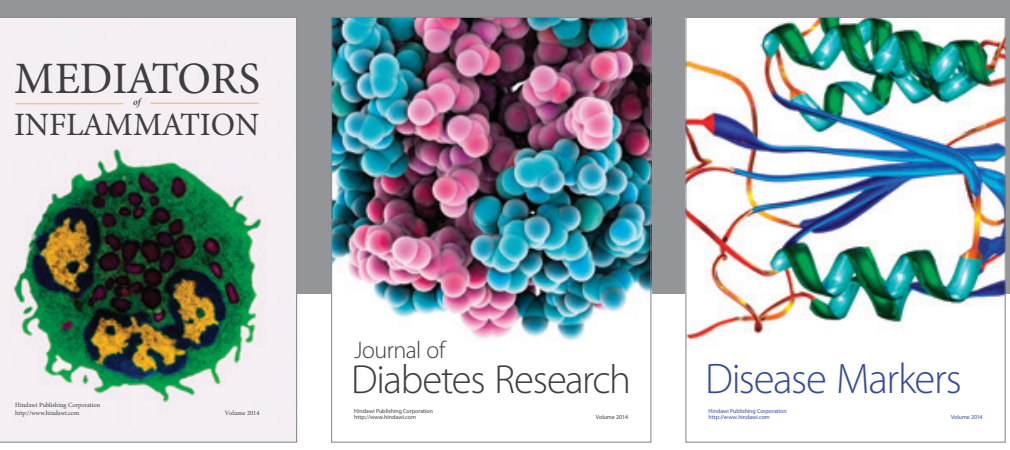

Disease Markers

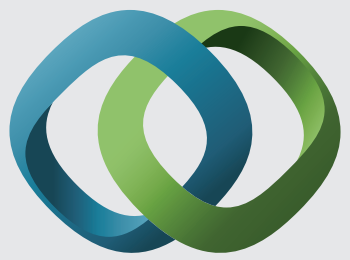

\section{Hindawi}

Submit your manuscripts at

https://www.hindawi.com
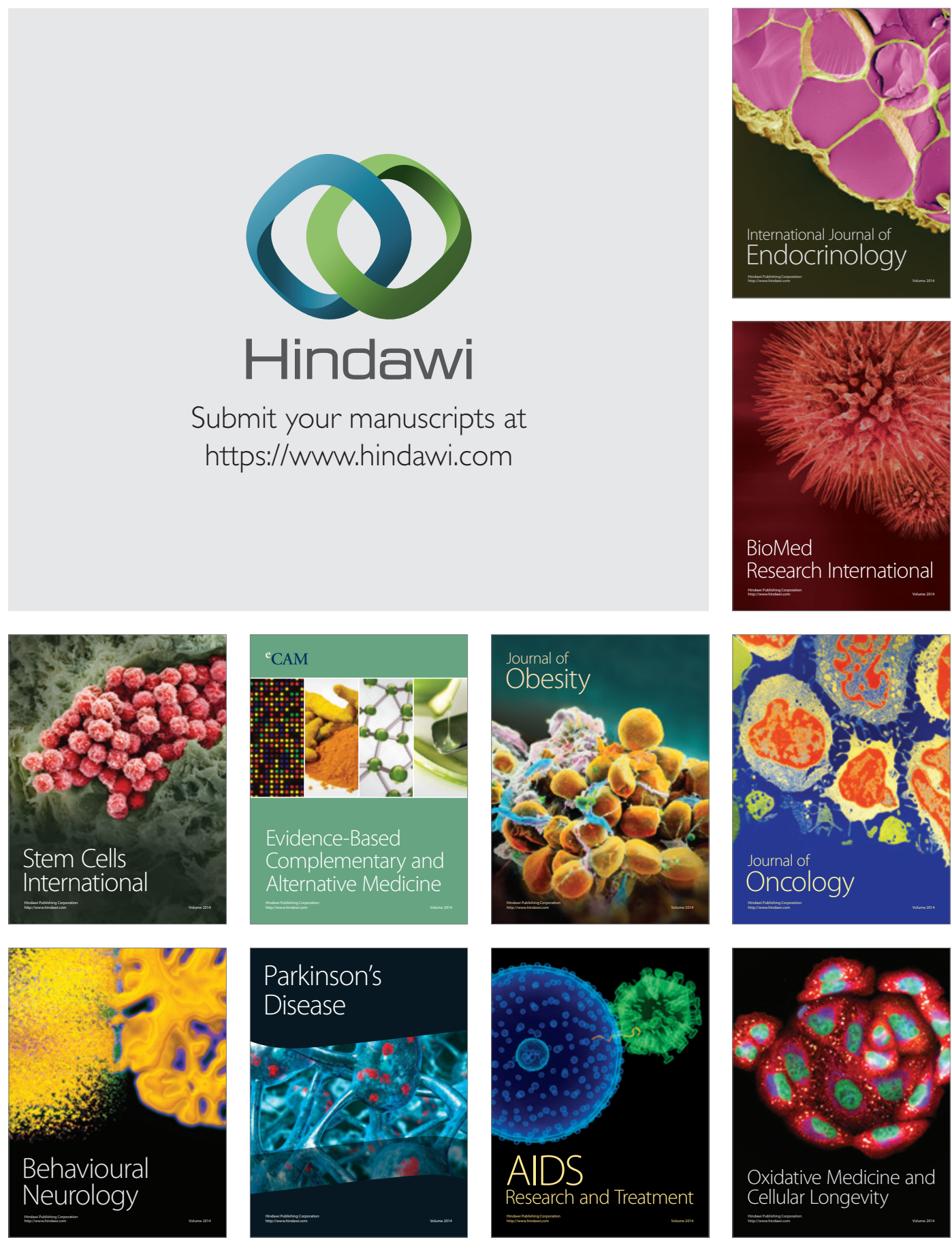